\title{
CONNECTION BETWEEN WAVE TRANSPORT THROUGH DISORDERED 1D WAVEGUIDES AND ENERGY DENSITY INSIDE THE SAMPLE: A maximum-entropy approach
}

\author{
Pier A. Mello ${ }^{\mathrm{a}, *}$, Zhou Shi ${ }^{\mathrm{b}}$, Azriel Z. Genack ${ }^{\mathrm{b}}$ \\ ${ }^{a}$ Instituto de Física, Universidad Nacional Autónoma de México, Apartado Postal \\ 20-364, 01000 México D.F., Mexico \\ ${ }^{b}$ Department of Physics, Queens College and the Graduate Center of the City University \\ of New York, Flushing, NY, 11367 USA
}

\begin{abstract}
We study the average energy - or particle - density of waves inside disordered 1D multiply-scattering media. We extend the transfer-matrix technique that was used in the past for the calculation of the intensity beyond the sample to study the intensity in the interior of the sample by considering the transfer matrices of the two segments that form the entire waveguide. The statistical properties of the two disordered segments are found using a maximum-entropy ansatz, subject to appropriate constraints. The theoretical expressions are shown to be in excellent agreement with 1D transfermatrix simulations.
\end{abstract}

Keywords: Random-matrix theory, wave propagation

\section{Introduction}

Studies of the scaling of the average transmission and the full probability distribution of transmission and conductance through disordered media at various degrees of spatial averaging over the output and input surface has played a central role in localization and mesoscopic physics $[1,2,3,4,5$, $6]$. Interest in the profiles of energy or particle density inside random open

\footnotetext{
*Corresponding author

Email address: mello@fisica.unam.mx (Pier A. Mello)
} 
samples, which has been of great current interest [7, 8, 9, 10, 11, 12], goes back even further. In the work of Ref. [13] a detailed calculation is reported in which successive averaging over impurities is performed to yield the result of Eq. (26) of that reference for the average intensity inside the sample. A further generalization of this result (allowing for impedance mismatch at the generator and the load, in the electric-circuit terminology used by the authors), which makes use of the notion of transfer matrices, is given in Ref. [14].

It is interesting to recall that for a classical system, the intensity $I(z)$ in the interior of a diffusing sample falls linearly within the sample, as required by Fick's law of particle diffusion; this yields a constant diffusion coefficient $D_{0}$. A first-order correction to the diffusion equation due to localization effects was obtained recently by introducing a one-loop weak localization correction together with the assumption of the self-consistency of this approximation $[15,16]$. This has yielded a generalized diffusion equation with a position-dependent diffusion coefficient, $D(x)$. This diffusion coefficient is increasingly renormalized with increasing depth into the sample by the destructive interference of waves returning to points inside the medium. Good agreement with this self-consistent theory [17] is obtained in simulations and in optical measurements in a multichannel slot in a periodic $2 \mathrm{D}$ structure. When extended to the time domain [10], the self-consistent theory gives good agreement [18] with the transmitted pulse profile for ultrasound in the localization transition. For more deeply localized samples, however, microwave transmission at long times was dramatically slowed down because of the increasing contribution of long-lived quasi-normal modes to transmission at late times [19]. An exact theory beyond the single-loop approximation has been developed based on the supersymmetry approach [20]. The problem of an inhomogeneous diffusion coefficient dependent on system size has been discussed recently in Ref. [21], based on the results of Ref. [13].

An alternative approach to the statistical properties of disordered conductors based on a maximum-entropy ansatz was presented in detail in Ref. [22], where an equation governing these properties, the Dorokhov-Mello-PereyraKumar (DMPK) equation, is derived using this method. In this approach, the disordered system is assumed to contain a large number of very weak scatterers: this was called the dense-weak-scattering limit. It is expected that in this limit the maximum-entropy ansatz will give results largely independent of the microscopic details. The transfer matrix is particularly useful in this context due to its multiplicative property. The result is thus 
a random-matrix theory (RMT) of disordered systems, in which one studies an ensemble of transfer matrices.

To the best of our knowledge, RMT has not been widely used to explore the statistics of propagation inside random media. One exception is Ref. [14] which, in the limit of uncorrelated disorder, finds an exact solution to the problem. Ref. [13] uses, instead, recursive relations and an induction method. Ref. [21] makes use of the results of Ref. [13] to compute the average intensity inside the sample.

In the present contribution, we develop an approach based on the DMPK equation mentioned above to find the average intensity profile inside random $1 \mathrm{D}$ samples. As we indicated, the method based on a maximum-entropy ansatz, although not exact, is expected to give results largely independent of the microscopic details. These calculations are in excellent agreement with computer simulations. In our opinion, the interest of the present point of view lies in the conceptual simplicity of the maximum-entropy approach, and the possibility of extending the analysis to other quantities of physical interest; it may provide an opening to calculate the average profile of energy density inside 1D or quasi-1D samples for transmission eigenchannels with specified values of transmission. These profiles were recently found in computer simulations [12]. The profiles were found to have a form related to the auxiliary localization lengths proposed by Dorokhov [5]. The structure of these profiles is consistent with the generalized diffusion equation with a position dependent diffusion coefficient and appropriately chosen source term and boundary conditions [12]. Since it is possible to manipulate the incident profiles of classical waves, the prospect exists of controlling the energy density inside random systems.

The paper is organized as follows. In Sec. 2 we construct an expression for the intensity inside a 1D sample, when incidence is from the left of the sample. Using the maximum-entropy ansatz outlined above, we average this result over an ensemble of disordered configurations to obtain our central result, Eq. (11a) below. Excellent agreement is found in a comparison of these results with computer simulations. Discussion of these results and perspectives for future research are presented in Sec. 3. In order not to interrupt the flow of the presentation in the main text, two appendices containing mathematical details have been added. 


\section{The intensity inside a $1 \mathrm{D}$ waveguide}

Consider the problem of scattering by a one-dimensional (1D) random distribution of scatterers, as illustrated in Fig. 1. This situation may arise in a Quantum Mechanical (QM) problem describing electronic scattering in a disordered conductor, or, more generally, in a wave-scattering problem in a disordered waveguide supporting a single transverse mode, or for a plane wave impinging upon a random layered medium. In what follows, we shall refer specifically to the first type of problem and use the QM nomenclature, although the notions from both fields can be used interchangeably.

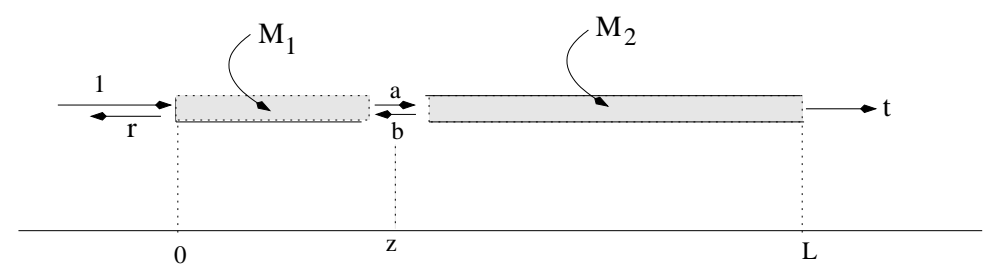

Figure 1: The scattering problem associated with the 1D disordered waveguide described in the text. The waveguide has length $L$ and can support one propagating mode, or channel. Indicated are the amplitudes of the incident, transmitted and reflected waves at either end of the waveguide. A small gap is opened at the point $z$ inside the sample, where the amplitudes of the waves travelling to the right and left are also shown. The transfer matrices $M_{1}, M_{2}$ of the two parts of the sample are also indicated.

The amplitude of the plane wave incident from the left is taken to be 1 ; the effect of the scattering process is to produce a reflected wave with amplitude $r$ on the left of the whole system, and a transmitted wave on the right, with amplitude $t$.

Inside the conductor, a distance $z$ from the left side of the sample, the wave function consists of a wave travelling to the right, with amplitude $a$, and a wave travelling to the left, with amplitude $b$, as also shown in Fig. 1. As described in the Introduction, the goal is to find the average intensity of the wave at the point $z$ inside the conductor.

We express the transfer matrices of the two portions of the waveguide as

$$
M_{i}=\left[\begin{array}{cc}
\alpha_{i} & \beta_{i} \\
\beta_{i}^{*} & \alpha_{i}^{*}
\end{array}\right], \quad i=1,2,
$$

with the condition $\left|\alpha_{i}\right|^{2}-\left|\beta_{i}\right|^{2}=1$, thus satisfying the requirements of timereversal invariance and flux conservation. When no index $i$ is employed, we shall understand the various quantities to refer to the wire as a whole. 
From the definition of the transfer matrix, we have

$$
M_{2}\left[\begin{array}{l}
a \\
b
\end{array}\right]=\left[\begin{array}{l}
t \\
0
\end{array}\right] .
$$

We invert this equation to find $a$ and $b$, making use of the relation

$$
M_{2}^{-1}=\left[\begin{array}{rr}
\alpha_{2}^{*} & -\beta_{2} \\
-\beta_{2}^{*} & \alpha_{2}
\end{array}\right],
$$

to find

$$
\left[\begin{array}{l}
a \\
b
\end{array}\right]=\left[\begin{array}{r}
t \alpha_{2}^{*} \\
-t \beta_{2}^{*}
\end{array}\right],
$$

where the transmission amplitude $t$ can be expressed as

$$
t=\frac{1}{\alpha^{*}}=\frac{1}{\alpha_{2}^{*} \alpha_{1}^{*}+\beta_{2}^{*} \beta_{1}} .
$$

The intensity inside the gap is then given by the equivalent expressions

$$
\begin{aligned}
I_{z}\left(M_{1}, M_{2}\right) & =\left|a e^{i k z}+b e^{-i k z}\right|^{2} \\
& =T\left|\alpha_{2}^{*} e^{i k z}-\beta_{2}^{*} e^{-i k z}\right|^{2} \equiv T F_{z}\left(M_{2}\right) \\
& =\frac{\left|\alpha_{2}^{*} e^{i k z}-\beta_{2}^{*} e^{-i k z}\right|^{2}}{\left|\alpha_{2} \alpha_{1}+\beta_{2} \beta_{1}^{*}\right|^{2}} .
\end{aligned}
$$

Using the polar representation defined in A.1, we can write the functions appearing in Eqs. (6) as

$$
\begin{aligned}
F_{z}\left(M_{2}\right)= & 1+2 \lambda_{2}-2 \sqrt{\lambda_{2}\left(1+\lambda_{2}\right)} \cos \left(2\left(\mu_{2}-\theta_{2}+k z\right)\right), \\
1 / T=1+ & \lambda_{1}+\lambda_{2}+2 \lambda_{1} \lambda_{2} \\
& +2 \sqrt{\lambda_{1} \lambda_{2}\left(1+\lambda_{1}\right)\left(1+\lambda_{2}\right)} \cos \left(2\left(\theta_{2}+\mu_{1}-\mu_{2}\right)\right) .
\end{aligned}
$$

The above expressions refer to one sample, i.e., to one configuration of disorder. Making the assumption of uncorrelated disorder, the various quantities referring to the two sections that form the full sample are statistically independent of one another. 
The average over an ensemble of configurations of the intensity $I_{z}\left(M_{1}, M_{2}\right)$ has to be computed with the probability distribution of the transfer matrices for the two sections of the waveguide; i.e.,

$$
\left\langle I_{z}\left(M_{1}, M_{2}\right)\right\rangle=\iint I_{z}\left(M_{1}, M_{2}\right) p_{z}\left(M_{1}\right) p_{L-z}\left(M_{2}\right) d \mu\left(M_{1}\right) d \mu\left(M_{2}\right) .
$$

As described in the Introduction, a maximum-entropy ansatz [22] yields, for the description of each section, as well as of the full sample, a DMPK equation for the "evolution" with length of the probability density of the "radial" parameters defined in the polar representation of the transfer matrix. For $N=1$ channel there is only one radial parameter $\lambda \geq 0$ (see Appendix A); denoting the probability density by $w_{s}(\lambda)$, the evolution equation becomes

$$
\frac{\partial w_{s}(\lambda)}{\partial s}=\frac{\partial}{\partial \lambda}\left[\lambda(1+\lambda) \frac{\partial w_{s}(\lambda)}{\partial \lambda}\right]
$$

known as the Melnikov equation [4]. Here, $s=L / \ell$ is the total length of the sample measured in units of the mfp.

The diffusion equation (9) has to be solved with the initial condition

$$
w_{s=0}(\lambda)=\delta_{+}(\lambda),
$$

$\delta_{+}(\lambda)$ denoting a one-sided delta function.

\subsection{The average $\langle I\rangle_{\zeta}$}

It is shown in Appendix $B$ that the calculation of the average intensity inside the conductor can be reduced to quadratures, consisting in the evaluation of the four-fold integral (see also Eq. (11c))

$$
\langle I\rangle_{\zeta}=1-\int_{0}^{\infty} \int_{0}^{\infty} g\left(\lambda_{1}, \lambda_{2}\right) w_{\zeta}\left(\lambda_{1}\right) w_{s-\zeta}\left(\lambda_{2}\right) d \lambda_{1} d \lambda_{2}
$$

the result depending on $\zeta=z / \ell$, the distance $z$ from the left edge of the sample measured in units of the $\operatorname{mfp} \ell$, and $s=L / \ell$. In Eq. (11a) we defined

$$
g\left(\lambda_{1}, \lambda_{2}\right)=\frac{\lambda_{1}-\lambda_{2}}{1+\lambda_{1}+\lambda_{2}}
$$

The function

$$
w_{s}(\lambda)=e^{-s / 4} \int_{0}^{\infty} f_{t}(\lambda) e^{-s t^{2}} 2 t \tanh (\pi t) d t
$$


is the probability density of the variable $\lambda$ for a $1 \mathrm{D}$ system of length $s=L / \ell$ (see Ref. [23], Eq. (5.58)) and is the solution of Melnikov's equation (9). We use the simplified notation

$$
f_{t}(\lambda)={ }_{2} F_{1}\left(\frac{1}{2}+i t, \frac{1}{2}-i t ; 1 ;-\lambda\right)
$$

to denote the usual hypergeometric function.

\subsubsection{Checks for some particular cases}

1) $\zeta=0$, i.e., just at the left end of the sample

$$
\begin{aligned}
w_{\zeta}\left(\lambda_{1}\right) & =w_{0}\left(\lambda_{1}\right)=\delta_{+}\left(\lambda_{1}\right) \\
w_{s-\zeta}\left(\lambda_{2}\right) & =w_{s}\left(\lambda_{2}\right) \\
\langle I\rangle_{0} & =1+\int_{0}^{\infty} \frac{\lambda_{2}}{1+\lambda_{2}} w_{s}\left(\lambda_{2}\right) d \lambda_{2} \\
& =1+\langle R\rangle_{s}
\end{aligned}
$$

Indeed:

$$
\langle I\rangle_{0}=\left\langle|1+r|^{2}\right\rangle_{s}=1+\langle R\rangle_{s} .
$$

2) $\zeta=L / \ell=s$, i.e., just at the right end of the sample

$$
\begin{aligned}
w_{\zeta}\left(\lambda_{1}\right) & =w_{s}\left(\lambda_{1}\right) \\
w_{s-\zeta}\left(\lambda_{2}\right) & =w_{0}\left(\lambda_{2}\right)=\delta_{+}\left(\lambda_{2}\right) \\
\langle I\rangle_{s} & =1-\int_{0}^{\infty} \frac{\lambda_{1}}{1+\lambda_{1}} w_{s}\left(\lambda_{1}\right) d \lambda_{1} \\
& =1-\langle R\rangle_{s}=\langle T\rangle_{s} .
\end{aligned}
$$

To check Eq. (11a), we have carried out simulations for a normally incident wave impinging on a layered sample embedded in air. The sample consists of alternating layers with refractive indices 1 and 1.5. The thickness of each layer is selected from a uniform distribution between 30 and 70 $\mu m$. The intensity inside the sample was calculated using scattering-matrix simulations at each wavelength over a spectrum from 0.4 to $0.9 \mu \mathrm{m}$ with a spacing of $0.01 \mu \mathrm{m}$. Since the layer thickness is much greater than the wavelength, the phase accumulated for a wave propagating through a single layer is randomized and the statistics at each wavelength are equivalent. The localization length of the sample, which is the same as the transport mfp $\ell$ in 

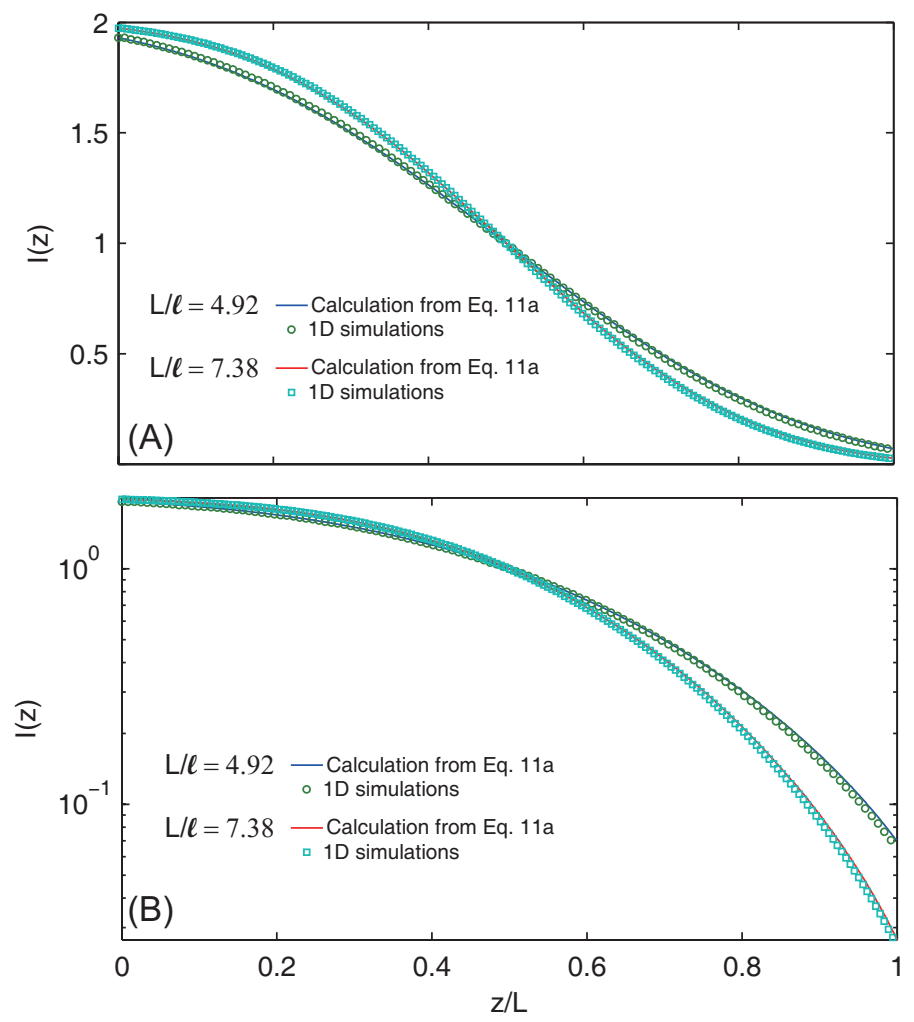

Figure 2: Comparison of the theoretical result of Eq. (11a) for the average intensity inside a disordered $1 \mathrm{D}$ system and computer simulations, for two values of $L / \ell$ (4.92 and 7.38), on a linear scale (A) and semi-log scale (B). The results are presented as function of $z / L, z$ being the distance from the left end of the sample and $L$ the total length. We recall that the value of $s$ used to compute Eq. (11a) is $L / \ell$, while $\zeta=z / \ell$. An excellent agreement is seen between the numerical calculations of Eq. (11a) and 1D simulations.

1D samples, is found to be 12.2 pairs of layers, by considering the scaling of the average of the logarithmic transmission. In Fig. 2 we present linear and semi-logarithmic plots of the average intensity $\langle I\rangle_{\zeta}=\langle I\rangle_{\frac{z}{L} s}$ as a function of position $z / L$ for two sample lengths, $s=L / \ell=4.92$ and 7.38 , as well as the numerical integration of Eq. (11a) with the same values of $s=L / \ell=4.92$ and 7.38. The average intensity in the simulations is obtained by averaging over the spectrum and over 100,000 sample realizations. Excellent agreement is found, confirming the validity of Eq. (11a).

We wish to point out that, when this paper was close to completion, it came to the authors' attention that Ref. [14] and the very recent Ref. [21] 
(which reports a calculation of the average intensity inside a 1D disordered system based on the theory of Ref. [13]) present in their Figs. 2-3 and 1a, respectively, results that are similar to our Fig. 2. The approach used in these references was briefly mentioned in the Introduction. For comparison we show in Fig. 3, together with the results of Fig. 2, those obtained from Eq. (7) of Ref. [21] for the same values of $L / \ell$; the latter, in turn, are based on the results of Ref. [13]. The three calculations are in excellent agreement.
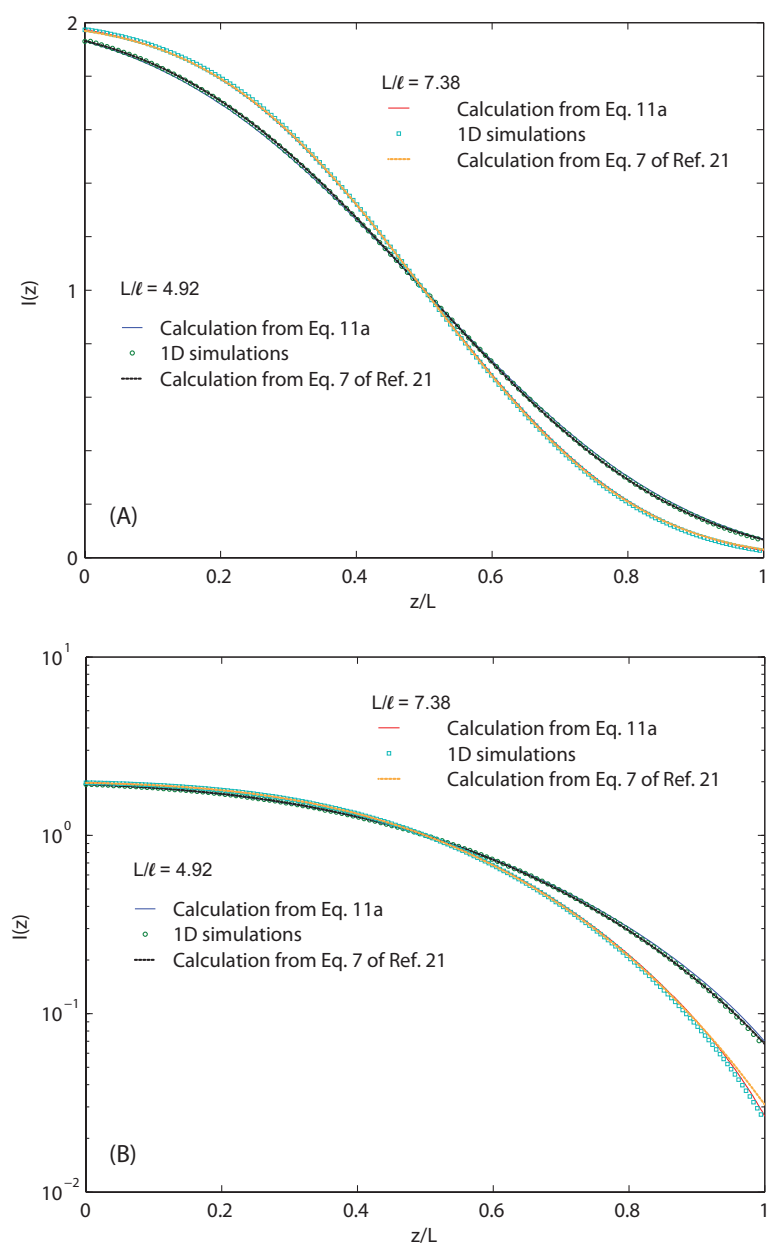

Figure 3: The average intensity as a function of $z / L$ obtained from Eq. (7) of Ref. [21] for the same values of $L / \ell$ as in Fig. 2, which is also shown for comparison. The results of i) Eq. (11a), ii) the computer simulations, and iii) Ref. [21] lie almost on top of each other. 


\section{Conclusions}

In this contribution we addressed the problem of the statistics of wave propagation inside a 1D disordered medium using random-matrix-theory techniques. In particular, we computed the average wave intensity inside the waveguide.

We approached the problem by proposing a maximum-entropy ansatz to find the statistical distribution of the transfer matrices associated with the sections of the waveguide on the left and right of the point of interest: this approach leads to a one-channel DMPK equation known as Melnikov's equation. The theoretical predictions for the average intensity profile are in excellent agreement with computer simulations.

In future investigations we plan to study a number of problems related to the statistics of transfer matrices: i) the average intensity profile conditioned on a given value of the total transmission coefficient for the full waveguide and ii) the extension of the description to an $N$-channel waveguide.

We have also computed the average intensity using Eq. (7) of Ref. [21], for the same values of $L / \ell$ used with our approach in Fig. 2. The agreement is excellent.

\section{Acknowledgments}

The authors are indebted to B. Shapiro for suggesting the derivation of Eq. (6c) through the steps starting from Eq. (2). PAM and AG wish to thank the Israel Institute of Technology (Haifa, Israel), where this work was started, for its hospitality. One of the authors (PAM) acknowledges support by DGAPA under contract No. IN109014, and the authors ZS and AZG by the National Science Foundation (DMR-1207446). The authors also wish to thank V. Gopar for pointing out a number of misprints in various equations. 


\section{Appendix A. The polar representation}

In a strictly $1 \mathrm{D}$ problem, the transfer matrix $M$ is a $2 \times 2$ matrix which can be parametrized as (Ref. [22], Eq. (7.35))

$$
\begin{aligned}
M & =\left[\begin{array}{cc}
e^{i \mu} & 0 \\
0 & e^{-i \mu}
\end{array}\right]\left[\begin{array}{rr}
\sqrt{1+\lambda} e^{i \theta} & \sqrt{\lambda} e^{-i \theta} \\
\sqrt{\lambda} e^{i \theta} & \sqrt{1+\lambda} e^{-i \theta}
\end{array}\right]\left[\begin{array}{cc}
e^{-i \mu} & 0 \\
0 & e^{i \mu}
\end{array}\right] \\
& =\left[\begin{array}{cc}
\sqrt{1+\lambda} e^{i \theta} & \sqrt{\lambda} e^{i(2 \mu-\theta)} \\
\sqrt{\lambda} e^{-i(2 \mu-\theta)} & \sqrt{1+\lambda} e^{-i \theta}
\end{array}\right]
\end{aligned}
$$

As the length of the system under consideration tends to zero, we have $\lambda \rightarrow 0$ and $\theta \rightarrow 0$, while $\mu$ remains arbitrary. In this limit, the statistical distribution of $M$ must develop appropriate delta functions to ensure the fulfilment of these conditions.

\section{Appendix B. Proof of Eq. (11a)}

In terms of the probability density $w_{s}(\lambda, \theta)$ defined in Ref. [22], we can write Eq. (8) as

$\left\langle I_{z}\left(M_{1}, M_{2}\right)\right\rangle=\int_{0}^{\infty} \int_{0}^{\infty} d \lambda_{1} d \lambda_{2} \int_{0}^{2 \pi} \int_{0}^{2 \pi} d \theta_{1} d \theta_{2} \bar{I}_{z}\left(\lambda_{1}, \theta_{1}, \lambda_{2}, \theta_{2}\right) w_{z}\left(\lambda_{1}, \theta_{1}\right) w_{L-z}\left(\lambda_{2}, \theta_{2}\right)$

where

$$
\begin{aligned}
\bar{I}_{z}\left(\lambda_{1}, \theta_{1}, \lambda_{2}, \theta_{2}\right) & =\int_{0}^{2 \pi} \int_{0}^{2 \pi} \frac{d \mu_{1}}{2 \pi} \frac{d \mu_{2}}{2 \pi} I_{z}\left(\lambda_{1}, \theta_{1}, \mu_{1} ; \lambda_{2}, \theta_{2}, \mu_{2}\right) \\
& =\int_{0}^{2 \pi} \int_{0}^{2 \pi} \frac{d \mu_{1}}{2 \pi} \frac{d \tilde{\mu}_{2}}{2 \pi} \frac{A+B \cos \left(2\left(\tilde{\mu}_{2}+\mu_{1}+k z\right)\right)}{C+D \cos \left(2 \tilde{\mu}_{2}\right)}
\end{aligned}
$$

The integrand in (B.2a), $I_{z}\left(\lambda_{1}, \theta_{1}, \mu_{1} ; \lambda_{2}, \theta_{2}, \mu_{2}\right)$, is a more explicit version of $I_{z}\left(M_{1}, M_{2}\right)$, and is taken from Eqs. (6) and (7); $A, B, C, D$ are given by

$$
\begin{aligned}
& A=1+2 \lambda_{2} \\
& B=-2 \sqrt{\lambda_{2}\left(1+\lambda_{2}\right)} \\
& C=1+\lambda_{1}+\lambda_{2}+2 \lambda_{1} \lambda_{2} \\
& D=2 \sqrt{\lambda_{1} \lambda_{2}\left(1+\lambda_{1}\right)\left(1+\lambda_{2}\right)} .
\end{aligned}
$$


In Eq. (B.2b) we made the change of variables $\tilde{\mu}_{2}=\mu_{2}-\mu_{1}-\theta_{2}$.

We thus find (see Ref. [24], p. 366, Eq. (3.613.1), for $|D / C|<1$ )

$$
\begin{aligned}
\bar{I}_{z}\left(\lambda_{1}, \theta_{1}, \lambda_{2}, \theta_{2}\right) & =\frac{A}{C} \frac{1}{2 \pi} \int_{0}^{2 \pi} \frac{d \phi}{1+\frac{D}{C} \cos \phi} \\
& =\frac{A}{\sqrt{C^{2}-D^{2}}}, \\
& =1-g\left(\lambda_{1}, \lambda_{2}\right),
\end{aligned}
$$

a result independent of $\theta_{1}$ and $\theta_{2}$, so that, in Eq. (B.1), $\int w_{z}\left(\lambda_{1}, \theta_{1}\right) d \theta_{1}=$ $w_{z / \ell}\left(\lambda_{1}\right)$, and similarly for $w_{L-z}$. Here, $g\left(\lambda_{1}, \lambda_{2}\right)$ is as defined in Eq. (11b).

Finally, Eq. (B.1) gives the desired result (11a).

\section{References}

\section{References}

[1] D. J. Thouless, "Metallic resistance in thin wires," Phys. Rev. Lett. 39, 1167 (1977).

[2] E. Abrahams, P. Anderson, D. C. Licciardello, and T. V. Ramakrishnan, "Scaling theory of localization: absence of quantum diffusion in two dimension," Phys. Rev. Lett. 42, 673 (1979).

[3] P. W. Anderson, D. J. Thouless, E. Abrahams, and D. S. Fisher, "New method for a scaling theory of localization," Phys. Rev. B 22, 3519 (1980).

[4] V. I. Mel'nikov, "Distribution of resistivity probabilities of a finite, disordered system," Pis'ma Zh. Ekps. Teor. Fiz. 32, 244 (1980) [JETP Lett. 32, 225 (1980)]; Fis. Tverd. Tela (Leningrad) 23, 782 (1981) [Sov. Phys. Solid State 23, 444 (1981)].

[5] O. N. Dorokhov, "On the coexistence of localized and extended electronic states in the metallic phase," Solid State Commun. 51, 381 (1984).

[6] M. C. W. van Rossum and T. M. Nieuwenhuizen, "Multiple scattering of classical waves: microscopy, mesoscopy, and diffusion," Rev. Mod. Phys. 71, 313 (1999). 
[7] W. Choi, A. P. Mosk, Q. Park, and W. Choi, "Transmission eigenchannels in a disordered medium," Phys. Rev. B 83, 134207 (2011).

[8] B. Gérardin, J. Laurent, A. Derode, C. Prada, and A. Aubry, "Full transmission and reflection of waves propagating through a maze of disorder," Phys. Rev. Lett. 113, 173901 (2014).

[9] S. Liew, S. Popoff, A. P. Mosk, W. L. Vos, and H. Cao, "Transmission channels for light in absorbing random media: from diffusive to ballisticlike transport," Phys. Rev. B 89, 224202 (2014).

[10] A. G. Yamilov, R. Sarma, B. Redding, B. Payne, H. Noh, and H. Cao, "Position-dependent diffusion of light in disordered waveguides," Phys. Rev. Lett. 112, 023904 (2014).

[11] M. Davy, Z. Shi, J. Wang, X. Cheng, and A. Z. Genack, "Transmission eigenchannels and the densities of states of random media," Phys. Rev. Lett. 114, 033901 (2015).

[12] M. Davy, Z. Shi, J. Park, C. Tian, and A. Z. Genack, "Universal structure of transmission eigenchannels inside opaque media," Nat. Commun. 6, 6893 (2015).

[13] Y. L. Gazaryan, "The one-dimensional problem of propagation of waves in a medium with inhomogeneities," Sov. Phys. 29, 996 (1969).

[14] W. Kohler and G. C. Papanicolau, "Power statistics for wave propagation in one dimension and comparison with radiative transport theory," J. Math. Phys. 14, 1733 (1973).

[15] B. A. van Tiggelen, A. Lagendijk, and D. S. Wiersma, "Reflection and Transmission of Waves near the Localization Threshold," Phys. Rev. Lett. 84, 4333 (2000).

[16] S. E. Skipetrov and B. A. van Tiggelen, "Dynamics of Anderson Localization in Open 3D Media," Phys. Rev. Lett. 96, 043902 (2006).

[17] B. Payne, A. Yamilov, and S. E. Skipetrov, "Anderson localization as position-dependent diffusion in disordered waveguides," Phys. Rev. B 82, 024205 (2010). 
[18] H. Hu, A. Strybulevych, J. H. Page, S. E. Skipetrov, and B. van Tiggelen, "Localization of ultrasound in a three dimensional elastic network," Nat. Phys. 4, 945 (2008).

[19] Z. Q. Zhang, A. A. Chabanov, S. K. Cheung, C. H. Wong, and A. Z. Genack, "Dynamics of localized waves: Pulsed microwave transmissions in quasi-one-dimensional media," Phys. Rev. B 79, 144203 (2009).

[20] C. Tian, S. Cheung, and Z. Zhang, "Local diffusion theory for localized waves in open media," Phys. Rev. Lett. 105, 263905 (2010).

[21] P. Neupane and A. G. Yamilov, "Applicability of the position-dependent diffusion approach to localized transport through disordered waveguides," Phys. Rev. B 92, 014207 (2015).

[22] P. A. Mello and N. Kumar, Quantum Transport in Mesoscopic Systems (Oxford University Press, 2010.)

[23] P. A. Mello, J. Math. Phys. 27, 2876 (1986)

[24] I. S. Gradshteyn and I. M. Ryzhik, Table of Integrals, Series, and Products (Academic Press, New York and London, 1965.) 